\title{
Information-Sharing Intention on Anonymous Social Media: the Role of Anonymity, Secrecy and Similarity
}

\author{
Yang Pan*, Zhichao Cheng, and Yiwen Xu \\ School of Economics and Management, Beihang University, Beijing 100191, China \\ *Corresponding Author
}

Keywords: Information-sharing intention, Anonymous, Secrecy, Similarity

\begin{abstract}
The goal of our study was to explore the relationship between anonymity, secrecy, similarity and information-sharing intention in the context of anonymous SNAs. An online survey was conducted using a Soul user sample (aged 18-30 years; 149 males, 126 females). The results showed that, the perceived secrecy and similarity was positively associated with information-sharing intention; however, no significant relationship was found between perceived anonymity and information-sharing intention. Theoretical implications and limitations are discussed.
\end{abstract}

\section{Introduction}

Anonymous social networking applications (SNAs) have become the main channel to meet the social needs of young people. In 2018, an anonymous SNAs called Soul launched and quickly became popular, especially on Chinese 90's and 00's. Soul not only allows users to modify ID information indefinitely, but also provides users with a variety of dating and matching channels to meet their differentiated social needs. Compared with the traditional system of random assignment, this personalized match pattern is easier to improve the probability of social relationship formation. Moreover, Soul constantly promotes hot topic discussion and personal behavior habits investigation, encourages users to share their experiences, views and insights. Users' information-sharing behavior plays an important role in the long-term and stable development of anonymous SNAs. However, few studies have explored the information-sharing behavior of anonymous SNAs users and its influencing factors. Therefore, the main purpose of the present study is to explore the impacts of anonymous SNAs features factors (e.g., anonymity, secrecy and interpersonal similarity) on users' information-sharing intention.

\section{Anonymity, Secrecy, Similarity and Information-Sharing}

In the post-Snowden era, anonymous SNAs have become an important choice for people to socialize online. In order to enrich the Internet life in the era of Web 2.0 and contribute real ideas and opinions, people need to remain anonymous. Anonymity is a strategy to hide one's identity. People may prefer to share content and information as anonymous online identities without worrying about the consequences of their actions. Some scholars believe that anonymity is a necessary tool to protect private information, freedom of expression, creativity and personal expression [1]. Anonymity enables people to hide or disclose information at will; as a core concept of information privacy, it is crucial to the ability to control personal information. Therefore, this study suggests that when users can hide their identity in anonymous social software, their willingness to share information may increase.

Secrecy is defined as intentionally hiding personal information, which allows users to avoid digital representations of their true selves [2]. People hide certain information because if others know it, it may lead to high risk. Therefore, people are likely to want to hide personal true information. When anonymous SNAs users can actively hide or even forge some personal information, they can maintain a high degree of control over the information. Therefore, this study 
suggests that when anonymous SNAs allows users to modify and hide personal information at will, users' willingness to share personal information may increase.

Similarity refers to, "the degree to which people who interact are similar in beliefs, education, social status, and the like" [3]. According to similarity extrapolation theory, similarity in preferences generates a positive affective reaction toward the other, which in turn motivates inferences of further similarity. When people interact with those who are similar to themselves, they engage in more emotional connections and feel easier and happier to communicate with each other in the online platform. Therefore, this study suggests that when anonymous SNAs provide users with the features of finding members with similar values, interests, hobbies and personalities, users' willingness to share information will be enhanced.

To summarize, we propose the following hypotheses:

H1. Perceived anonymity has a positive effect on users' behavioral intentions to sharing their personal information in the anonymity SNAs.

H2. Perceived secrecy has a positive effect on users' behavioral intentions to sharing their personal information in the anonymity SNAs.

H3. Perceived similarity has a positive effect on users' behavioral intentions to sharing their personal information in the anonymity SNAs.

\section{Method}

\subsection{Participants.}

A total of 275 Soul users (aged 18-30; 45.8\% females and 54.2\% males) through a survey website (www.wjx.cn) completed an online survey. Soul users who had registered on this sites and used Soul for at least 3 months were randomly invited to participate in this survey.

\subsection{Measures.}

Perceived anonymity and secrecy. We used Dinev et al (2013) 6-item (3 each), 5-point (1=strongly disagree; $5=$ strongly agree) scale to measure participants' perceptions of anonymity and secrecy on Soul [4].

Perceived similarity. We used Fu et al (2018) 4-item, 5-point (1=strongly disagree; 5=strongly agree) scale to measure participants' perceptions of interpersonal similarity on Soul [5].

Information-sharing intention. We used Chen et al (2019) 4-item, 5-point (1=strongly disagree; $5=$ strongly agree) scale to measure participants' perceptions of information-sharing intention on Soul [6].

Control variables. These consist of the number of gender, age and the amount of time spent on Soul per days.

\section{Results}

Table 1 Displays Descriptive Statistics of Each Variables and Bivariate Correlations with Them. the Data in Parentheses Represent the Cronbach A of Each Subscale.

Table 1 Means, Standard Deviations and Correlations among the Major Variables

\begin{tabular}{|l|l|l|l|l|l|l|}
\hline Variables & M & SD & 1 & 2 & 3 & 4 \\
\hline 1. Anonymity & 3.39 & 0.59 & $(0.77)$ & & & \\
\hline 2. Secrecy & 3.35 & 0.74 & -0.01 & $(0.69)$ & & \\
\hline 3. Similarity & 3.18 & 0.87 & 0.09 & $0.12^{*}$ & $(0.89)$ &. \\
\hline 4. Information-sharing & 3.18 & 0.95 & 0.11 & $0.18^{* *}$ & $0.41^{* *}$ & $(0.87)$ \\
\hline
\end{tabular}

Note: ${ }^{*} \mathrm{p}<0.05,{ }^{* *} \mathrm{p}<0.01,{ }^{* * *} \mathrm{p}<0.001$

The results of hierarchical multiple regression analyses are presented in Table 2. The control variables (gender, age and Soul usage) were entered as the first block of predictors, and anonymity, secrecy and similarity were added as the second block. Perceived secrecy and similarity were found to have a significant, positively effect on information-sharing intention indicating these features are 
important predictors of information-sharing intention on Soul, thus supporting hypothesis 2 and hypothesis 3. However, perceived anonymity has no significant positive effect on information-sharing intention on Soul, which is inconsistent with the hypothesis 1.

Table 2 Regression Information-Sharing Intention on Anonymity, Secrecy and Similarity

\begin{tabular}{|l|l|l|l|l|}
\hline \multicolumn{4}{|l|}{ Information-sharing intention } & \multicolumn{1}{l|}{} \\
\hline & $\mathrm{B}(\mathrm{SE})$ & $\beta$ & $\mathrm{R}^{2}$ & $\Delta \mathrm{R}^{2}$ \\
\hline Variables/steps & & & & \\
\hline Step1 & & & & \\
\hline Gender & 0.06 & 0.03 & & \\
\hline Age & 0.04 & 0.03 & & \\
\hline Usage time & 0.14 & $0.15^{*}$ & & \\
\hline $\mathrm{F}(3,271)$ & & & 0.02 & 0.02 \\
\hline Step2 & & & & \\
\hline Anonymity & 0.09 & 0.05 & & \\
\hline Secrecy & 0.17 & $0.13^{*}$ & & \\
\hline Similarity & 0.43 & $0.39^{* * *}$ & & $0.19^{* * * *}$ \\
\hline F(3, 268) & & & 0.21 & \\
\hline
\end{tabular}

Note: Gender was coded as 0 for female and 1 for male; ${ }^{*} \mathrm{p}<0.05,{ }^{* *} \mathrm{p}<0.01,{ }^{* * *} \mathrm{p}<0.001$.

\section{Discussion}

The goal of our study was to explore the relationship between anonymity, secrecy, similarity and information-sharing intention in the context of anonymous SNAs. Based on the results of multiple regression analysis, information-sharing intention was predicted by two features: secrecy and similarity. The result of the former shows that providing users with a good confidentiality environment and hiding their true selves will help to improve users' willingness to share information. The result of the latter shows that interpersonal similarity could increase users' willingness to trust members of the the anonymous SNAs and further increase the intentions for sharing personal information. However, the results of the present study did not show a significant positive effect of perceived anonymous on information-sharing intention. This shows that anonymous SNAs, like other acquaintances social media (e.g., WeChat), still need users to identify themselves, so as to facilitate further information-sharing. A close connection between the subjects of sharing behavior helps to cultivate the construction of hyper-personal impressions.

This study was limited in several ways. This study used a limited sample that certainly does not represent the all Soul population. Moreover, given the limitations in time and resources, cross-sectional studies are used as exploratory vehicles that can determine the relationships among variables. Finally, the influence of perceived anonymity, secrecy, similarity on information-sharing behavior still needs to be examined in more anonymous SNAs contexts.

\section{References}

[1] Brazier, F., Oskamp, A., Prins, C., Schellekens, M., \& Wijngaards, N. (2004). Anonymity and software agents: an interdisciplinary challenge. Artificial Intelligence and Law, 12(1-2), 137-157.

[2] Zwick, D., \& Dholakia, N. (2004). Whose identity is it anyway? Consumer representation in the age of database marketing. Journal of Macromarketing, 24(1), 31-43.

[3] Rogers, E. M., \& Bhowmik, D. K. (1970). Homophily-heterophily: Relational concepts for communication research. Public opinion quarterly, 34(4), 523-538.

[4] Dinev, T., Xu, H., Smith, J. H., \& Hart, P. (2013). Information privacy and correlates: an empirical attempt to bridge and distinguish privacy-related concepts. European Journal of Information Systems, 22(3), 295-316.

[5] Fu, S., Yan, Q., \& Feng, G. C. (2018). Who will attract you? Similarity effect among users on online purchase intention of movie tickets in the social shopping context. International Journal of 
Information Management, 40, 88-102.

[6] Chen, X., Sun, M., Wu, D., \& Song, X. Y. (2019). Information-Sharing Behavior on WeChat Moments: The Role of Anonymity, Familiarity, and Intrinsic Motivation. Frontiers in Psychology, 10, 2540. 